\title{
س
}

$>\mathrm{DE}$

$\simeq$ PÉDAGOGIE

\author{
Recherches en éducation
}

176 | juillet-septembre 2011

Varia

\section{Apprendre à être parent à la maternité : transmission et concurrence des savoirs}

Learning to be a parent at maternity hospital: Transmission and competition of knowledge

Aprender a ser padres en la maternidad: Transmisión y competencia de los conocimientos

Eltern werden im Entbindungsheim lernen: Vermittlung und Konkurrenz von

Wissen

Jérôme Camus et Nathalie Oria

\section{OpenEdition}

Journals

Édition électronique

URL : http://journals.openedition.org/rfp/3171

DOI : $10.4000 /$ rfp.3171

ISSN : 2105-2913

Éditeur

ENS Éditions

Édition imprimée

Date de publication : 15 septembre 2011

Pagination : 73-82

ISBN : 978-2-84788-329-9

ISSN : 0556-7807

Référence électronique

Jérôme Camus et Nathalie Oria, « Apprendre à être parent à la maternité : transmission

et concurrence des savoirs », Revue française de pédagogie [En ligne], 176 | juillet-septembre 2011, mis en ligne le 15 septembre 2015, consulté le 19 avril 2019. URL : http://journals.openedition.org/ rfp/3171; DOI : 10.4000/rfp.3171 


\title{
Apprendre à être parent à la maternité : transmission et concurrence des savoirs
}

\author{
Jérôme Camus et Nathalie Oria
}

L'encadrement de la grossesse et de la naissance s'opère en France grâce à un dispositif institutionnel traversé par des normes contrastées. Si le savoir médical domine, il est concurrencé depuis les années soixante-dix par les discours d'inspiration psychanalytique. Portée par différents corps professionnels (médecins, sages-femmes, auxiliaires de puériculture), cette tension se traduit aujourd'hui dans la division du travail d'encadrement et dans l'adaptation des pratiques des professionnels aux différentes catégories de mères.

Mots-clés (TESE) : apprentissage informel, personnel médical, relations parents-enfant, formation pratique, puériculture, situation sociale.

Le moment de la naissance fait l'objet d'un encadrement institutionnel intense, depuis l'annonce de la grossesse (visites prénatales), pendant l'accouchement (prise en charge par les sages-femmes, les médecins obstétriciens), lors du séjour en maternité, mais aussi lors du suivi post-maternité (sages-femmes, protection maternelle et infantile, médecins pédiatres). Au-delà de sa vocation proprement sanitaire, l'enjeu de cet encadrement consiste, à travers des techniques et des principes d'action, à circonscrire les manières légitimes selon lesquelles il convient de s'occuper d'un enfant jeune ou très jeune ${ }^{1}$. En tentant de les faire intérioriser, l'institution se voit chargée de définir et de transformer les pratiques associées au statut de parent (Donzelot, 1977). Le séjour à la maternité, parce qu'il concerne aujourd'hui la quasi-totalité des femmes qui accouchent en France ${ }^{2}$, constitue un terrain privilégié pour analyser comment s'opère cet encadrement institutionnel de l'accès au statut de parent. Pour envisa- ger comment l'institution hospitalière contribue à transmettre ces savoirs et ces pratiques associées au statut de parent et comment les hommes et les femmes qui vont ou viennent d'avoir un enfant réussissent (ou non) à s'en saisir, on peut prendre appui sur les nombreuses recherches abordant ces questions à propos de l'institution scolaire. Dans cette perspective, il s'agit ici d'interroger les rapports entre, d'une part, le milieu social d'origine, les trajectoires sociales et, d'autre part, les modalités d'action de l'institution et les rapports que ses usagers entretiennent avec elle.

Du côté de l'institution, il faut sans doute d'emblée indiquer qu'elle est marquée par une certaine hétérogénéité, tant du point de vue de l'intensité du contrôle social que des savoirs transmis. En effet, bien que s'incarnant dans des dispositifs relativement stabilisés, elle est traversée par les débats qui entourent le nouveau-né et le jeune enfant (Delaisi de Parseval 
\& Lallemand, 1979) : la montée des normes pastoriennes promues par les médecins qui, depuis la fin du $\mathrm{XIX}$ siècle, se donnaient pour but de lutter contre la mortalité infantile (Boltanski, 1969) se voit, depuis les années soixante-dix, remise en cause par les tenants d'une vision psychologique et psychanalytique, visant la préservation du «bon » développement de l'enfant par la mise à distance de prescriptions trop rigides ${ }^{3}$. Cette tension se retrouve aujourd'hui au sein même de l'hôpital, à l'intérieur duquel cohabitent différents corps professionnels plus ou moins enclins à s'approprier et à promouvoir ces différents types de savoirs. L'encadrement des parents s'inscrit dans cette tension, notamment à travers la délimitation différenciée au sein de l'institution des façons de "prendre soin " d'un nouveau-né et de leurs modes de transmission. Pour reprendre les analyses proposées par Grignon à propos de l'enseignement professionnel (Grignon, 1971), elle génère une action institutionnelle dédoublée, sous la forme d'un " discours fort " et d'un " discours faible ": les injonctions rationnelles et médicales, constitutives du " discours fort " que l'on trouve dans la définition officielle de la fonction de l'institution hospitalière, si elles sont reprises par l'ensemble des professionnels, sont ainsi tempérées par un ensemble de prescriptions concurrentes. À la rationalité visant la santé biologique s'oppose alors un « discours faible » reposant sur une éthique de la relation qui, associant l'idée de santé à celle de bien-être, promeut une vision de l'usager non plus seulement comme patient mais comme individu, comme personne «épanouie ».

Concernant les usagers de l'institution, c'est la question des rapports à ces savoirs concurrents et à leur appropriation qui se trouve posée. Les travaux de Séverine Gojard montrent que la famille revêt une importance grandissante du point de vue de la transmission des façons de s'occuper d'un nouveau-né, à mesure que l'on se déplace vers les catégories populaires (Gojard, 1998). Se rappelle ainsi l'importance de la famille comme instance de socialisation, notamment dans les rapports contrastés qui s'élaborent par rapport à l'école ou aux autres institutions d'encadrement (Willis, 1978 ; Schwartz, 1990 ; Thin, 1998), mais aussi le caractère socialement situé de l'éducation familiale (Pourtois, Desmet \& Lahaye, 2004 ; Kellerhals, Montandon, Gilbert et al., 1992), qui conduit à s'interroger sur la façon dont varie le rapport que les agents (ici les parents) entretiennent avec les différents professionnels au cours de leur séjour à la maternité. Par ailleurs, nombre de travaux sur les apprentissages scolaires (cf. par exemple Bonnéry, 2007) ou sur la transmission des savoirs médicaux (voir par exemple DurifBruckert, 2006) indiquent qu'avec la position sociale, ce sont les rapports aux savoirs et à leurs modes de transmission qui varient.

L'analyse des formes de transmission des savoirs entourant le nouveau-né, de leurs conditions sociales de réception par des agents situés en différents points de l'espace social conduit ainsi à interroger l'espace des savoirs associés au statut de parent. Dans cette perspective, Mauger et Poliak, en développant les analyses de Delbos et Jorion (1990), proposent, à partir d'une réflexion portant sur les pratiques de lecture, une analyse de l'espace social des types de savoirs (Mauger \& Poliak, 2000). À une hiérarchie sociale verticale qui décline des savoirs «pratiques", «techniques » et «théoriques ", ils associent une division plus horizontale, selon laquelle s'opposent le « monde des choses matérielles " et le "monde des choses humaines ". Cette inscription des différents types de savoirs dans les principes organisateurs de l'espace social permet d'envisager à la fois les mécanismes qui organisent les savoirs parfois contradictoires que tentent de transmettre, selon des modalités contrastées (" discours fort » ou " discours faible »), l'institution hospitalière, et les rapports de plus ou moins grande proximité sociale qu'entretiennent les agents avec les injonctions explicites ou implicites de l'institution. On verra ainsi, dans un premier temps, comment les hiérarchies professionnelles entre médecins, sagesfemmes et auxiliaires de puériculture redoublent, à l'intérieur du service, cette hiérarchie sociale des savoirs. En analysant les modes de transmission des pratiques parentales à la maternité, on envisagera ensuite les déclinaisons de l'action de l'institution sur ses usagers. Pour comprendre les effets très variables que peut avoir le séjour à la maternité sur l'intériorisation des façons d'être parent, on questionnera enfin, du côté des mères, la distribution inégale des dispositions à apprendre acquises au cours de leur trajectoire personnelle.

S'agissant d'appréhender la transmission des savoirs parentaux à la maternité, l'enquête sur laquelle on s'appuiera ici a été élaborée de façon à pouvoir rendre compte, d'une part, des pratiques des différentes catégories de professionnels et, d'autre part, du rapport des usagers à l'institution et à la naissance. C'est dans cette double perspective que les investigations ont été développées. D'abord, on a procédé à une observation du travail quotidien des personnels en privilégiant ceux dont la fonction participe de ce travail de transmission : auxiliaires de puériculture, sagesfemmes et médecins (en suivant "le tour [des chambres] »), notamment les internes. L'action des infirmières-puéricultrices dont une bonne partie du tra- 
vail consiste, dans les services étudiés, à informer le dossier médical et administratif, des aides-soignants ou des agents des services hospitaliers a fait l'objet d'observations plus ponctuelles. Par ailleurs, on a réalisé des entretiens (une quarantaine) à la maternité, avec des femmes (et plus rarement des hommes) venant d'avoir leur premier enfant. II s'agissait, à cette occasion, d'interroger l'expérience de la grossesse mais aussi la trajectoire antérieure et les représentations de l'avenir de ces mères.

Concernant les terrains d'enquête, deux maternités picardes ont été sélectionnées en fonction de leur taille, des moyens techniques dont elles disposent et du contexte socio-économique de leur espace d'implantation, de façon à pouvoir articuler les contextes organisationnels d'exercice des professionnels et à élargir le recrutement social des personnes interrogées. La première maternité, située à Amiens, est le principal centre de gynécologie et d'obstétrique de Picardie. Seule maternité de niveau $3^{4}$ de la Somme, plus de 2000 enfants y voient le jour tous les ans. À raison d'une à deux journées par semaine, les recherches y ont eu lieu pendant six mois. Réalisant 700 accouchements par an, la seconde maternité, de niveau 1, se trouve dans une ville de moyenne importance de l'Aisne, qui a connu récemment une forte désindustrialisation. Deux séquences d'enquête de plusieurs jours y ont été réalisées par un groupe de chercheurs de façon à pouvoir envisager les différentes dimensions de l'activité hospitalière autour de la naissance : service post-natal, salle de naissance, entretiens avec les parents et les professionnels ${ }^{5}$.

\section{HIÉRARCHIE PROFESSIONNELLE ET ENCADREMENTS INSTITUTIONNELS DES PARENTS}

L'un des enjeux du suivi des familles pendant la grossesse et pendant le séjour à la maternité consiste à transmettre des savoirs estimés nécessaires au bienêtre et au bon développement de l'enfant par les professionnels de l'institution. Cette prise en charge des familles constitue un moment d'éducation des futurs parents, pendant lequel des professionnels de la santé encouragent l'émergence chez les parents des comportements jugés adéquats et propices au bon développement de leur enfant. Comme le montre Pourtois, ces apprentissages sont centrés sur les pratiques éducatives quotidiennes (Pourtois, Forgione, Desmet et al., 1984).
La grossesse fait ainsi l'objet d'un contrôle, en particulier en milieu hospitalier ${ }^{6}$. Les futures mères sont confrontées à l'institution hospitalière, y sont totalement prises en charge, physiquement mais aussi socialement. Elles bénéficient d'un suivi, ainsi que de cours de préparation à la naissance. Lors de leur première visite prénatale, les futurs parents reçoivent systématiquement un " livre bleu7 » édité par le Comité national de l'enfance, opuscule censé servir de référence afin de répondre de manière « scientifique » aux questions qu'ils pourraient se poser sur la grossesse, la naissance et enfin sur l'éducation de l'enfant de 0 à 2 ans. Par ailleurs, tout au long de la grossesse, depuis sa découverte jusqu'à l'accouchement et lors des nombreux examens médicaux qui jalonnent la vie des parturientes, l'encadrement médical et paramédical est l'occasion de transmettre à la mère les « bons comportements " à adopter lorsqu'elle est enceinte. Ainsi, dès le premier rendez-vous gynécologique, la mère se voit proposer les normes de vie des futures mères (ne pas fumer, ne pas boire d'alcool, limiter les " comportements à risque »), normes justifiées par des arguments médicaux portant sur la santé de l'embryon et son développement. Béatrice Jacques remarque dans son ouvrage que « la première consultation chez le gynécologue permet un premier rappel ou apprentissage des règles socialement prescrites, reprises par la suite par les magazines et ouvrages spécialisés et par l'entourage » (Jacques, 2007). Dès le début de leur grossesse, puis de façon régulière jusqu'à l'accouchement, les femmes enceintes sont ainsi mises en situation d'apprentissage. Celui-ci a lieu de façon institutionnalisée lors des cours de préparation à la naissance ${ }^{8}$. Préparant les femmes à devenir de "bonnes " mères et de «bonnes " patientes, ils sont dispensés par des sages-femmes. Ces cours mobilisent des savoirs techniques (anatomie, déroulement physiologique et technique de l'accouchement, organisation de l'hôpital), transmis de façon magistrale, mais sont aussi l'occasion de mettre en avant, de manière plus empathique et dialoguée, les éléments selon lesquels doit s'établir la « qualité de la relation mère-enfant " : rôle du conjoint, séquences spécifiques incluses dans la procédure de naissance, comme la dépose sur le ventre ou la «tétée d'accueil ».

Cette activité pédagogique a donc bien pour but de mettre en concordance les dispositions sociales des futurs parents et les attentes de l'institution (Bourdieu, 1997). Elle est cependant structurée par les hiérarchies qui organisent les différents corps professionnels participant. Chaque profession tend ainsi à s'approprier certains types de savoirs, d'inspiration médicale ou psychologique, rationnelle ou relationnelle, selon l'his- 
toire de la division du travail propre au champ médical et paramédical et en s'inscrivant dans l'articulation sociale générale de différents registres de savoirs. Située au sommet de la hiérarchie des professions de santé intervenant dans le champ de l'encadrement de la maternité, la profession de médecin-gynécologue a beaucoup évolué depuis trente ans et cette évolution retentit sur les relations entre les patientes et le médecin. Longtemps située en bas de la hiérarchie médicale, la gynécologie a progressivement conquis ses lettres de noblesse grâce au développement de la recherche dans le domaine de l'embryologie, des progrès techniques en matière d'imagerie et d'anesthésie (Dagnaud \& Mehl, 1988 ; Jaisson, 2002). Les gynécologues-obstétriciens se chargent généralement du suivi des grossesses pathologiques, laissant aux sagesfemmes le soin d'accompagner les grossesses " normales ". Comme en témoignent les entretiens réalisés, les médecins se montrent souvent distants avec leurs patientes, asseyant leur autorité sur leur pouvoir social et sur leur compétence technique, utilisant un vocabulaire médical pointu, rechignant à donner des explications aux profanes, décourageant souvent les questions des patientes les plus angoissées (Cicourel, 2002) : «En fait, tu lui demandes des trucs et tout ça, mais après le médecin, il t'houspille un peu, c'est un quart d'heure pis après tu vires. Le médecin il t'explique pas, il s'occupe du médical quoi. " (femme de 29 ans, un enfant de 10 mois, licence de sociologie, employée).

En cela, ces médecins se différencient des sagesfemmes qui insistent plus souvent, selon la division horizontale mise en évidence par Mauger et Poliak (2000), sur la dimension " relationnelle » du suivi des grossesses. Ces dernières, en effet, occupent une place particulière dans l'institution : profession médicale à compétences limitées, elles doivent, selon leur référentiel de formation" ${ }^{9}$ " assurer la surveillance clinique et para-clinique de la grossesse ", mais aussi " dépister les situations à risque médical, psychique ou social et orienter alors les patientes vers un niveau de soin adapté ». Si cet objectif est primordial, elles doivent aussi « participer à la sécurité physique et psychologique de la mère et du nouveau-né à la naissance ", " assurer [leur] prise en charge globale » et " favoriser la relation mère-père-enfant ». Elles interviennent avant l'accouchement (en dispensant des cours de préparation et en assurant le suivi des grossesses non pathologiques), pendant l'accouchement puis pour les suites de couches, mais aussi par exemple en dispensant des cours d'allaitement. Ces professionnelles se spécialisent en effet traditionnellement dans le versant relationnel du suivi des femmes enceintes et des jeunes mères, remplissant en cela quelques-uns des objectifs de leur formation : "Accueillir et assurer les soins des nouveau-nés", "participer à la sécurité physique et psychologique de la mère et du nouveau-né à la naissance ", " assurer la prise en charge globale de la mère et de l'enfant " et «favoriser la relation mère-père-enfant. » Ce positionnement dans le registre du care s'éclaire donc lorsque l'on replace cette profession dans le champ de la maternité, au sein de l'institution hospitalière. Dominées par des médecins qui sont parvenus à construire en peu d'années une spécificité professionnelle quasiment idéal-typique, les sages-femmes luttent pour conserver une place légitime, oscillant entre hyper-technicité et valorisation du " bien-être ". Par exemple, les observations en salle d'accouchement mettent en évidence la volonté des sagesfemmes de procéder aux accouchements sans intervention des médecins. Lorsqu'une difficulté se présente lors du travail, le recours à l'obstétricien n'intervient qu'en dernière extrémité. II s'agit alors bien de protéger son espace de travail en utilisant sa capacité de définir le normal et le pathologique. Les savoirs qu'elles dispensent aux femmes enceintes dans les situations de face-à-face se présentent donc comme scientifiques, mais elles le transmettent de façon plus dialoguée que les médecins, n'hésitant pas à interroger les femmes pour s'assurer qu'elles ont bien compris certains points jugés importants et répondant aux questions posées afin d'établir une relation de confiance avec leurs patientes ${ }^{10}$.

Après l'accouchement, un troisième type de professionnels participe de façon privilégiée à la transmission des savoirs entourant le nourrisson : les auxiliaires de puériculture. Situées dans les positions les plus dominées de la hiérarchie professionnelle, elles se voient confier les tâches de gestion du quotidien des nouveau-nés. On note dans leur référentiel de formation la compétence suivante: "Accompagner l'enfant dans les activités d'éveil et de la vie quotidienne et les parents dans leur rôle éducatif ". En conséquence, les auxiliaires de puériculture doivent, en complémentarité avec les parents, apporter à l'enfant des « repères ", une « sécurité affective et physique » qui va l'encourager “ à grandir et à être autonome ». Concrètement, elles interviennent lors de la toilette, de l'habillage, des repas, de l'élimination et de la motricité et jouent un rôle éducateur en faisant respecter " les règles d'hygiène, de pudeur et de sécurité ». Enfin elles doivent accompagner et conforter les parents dans leur rôle éducatif, les associer aux soins et développer leurs capacités dans ce domaine, bref, les mettre sur la « voie de l'autonomie » pour préparer le retour à la 
maison. II s'agit donc de mobiliser des savoirs issus de la pratique auxquels l'institution confère une légitimité (Arborio, 2002). Toutes ces tâches s'effectuent sous forme de travaux pratiques, en plusieurs phases, les auxiliaires réalisant elles-mêmes, sous le regard des nouvelles mères, les soins aux nouveau-nés les premiers jours, puis en observant les nouvelles mères, en les encourageant et au besoin en les corrigeant les jours suivants.

\section{DES MODALITÉS PÉDAGOGIQUES AJUSTÉES}

Si tout au long de la grossesse, la division des savoirs et de leurs modes de transmission est ainsi soutenue par les hiérarchies professionnelles, c'est au moment de la naissance et dans les jours qui suivent, alors que les femmes se trouvent à l'intérieur de l'institution, qu'elle se donne à voir le plus clairement. Parce qu'il constitue un moment de mise en marge des nouvelles mères et de leur enfant (Tillard, 2003 , Carrière 2008), le séjour à la maternité permet d'analyser les modalités du travail d'imposition des normes entourant le nouveau-né, qui s'exerce alors de façon continue et avec plus de force qu'à aucun autre moment.

À un premier niveau, que l'on pourrait qualifier d'institutionnel, la légitimité rationnelle des savoirs médicaux impose des prescriptions techniques visant la bonne santé des patients. Ce « discours fort " est repris par l'ensemble des professionnels et gît dans les aspects matériels dont il a présidé à l'organisation ${ }^{11}$ : la séparation des lieux dédiés au repos et aux soins, les normes d'hygiène, mais aussi le matériel mis à disposition des parents (gant jetable, savon hypoallergénique, compresse stérile, désinfectant...), ou encore la décoration faite, pour une très large part, de photographies d'enfants au sein ou d'affiches de prévention, tout rappelle dans les maternités observées les normes devant être respectées par les mères pour garantir leur propre santé quotidienne et celle de leur enfant. Mais à un second niveau, ces injonctions rationnelles et médicales se voient tempérées par un « discours faible » qui, d'inspiration psychologique ou psychanalytique, vise le « bien-être » et l'« épanouissement » de la mère et de son enfant ${ }^{12}$. Cette articulation des discours, qui prend appui sur la distribution sociale des savoirs, se retrouve à tous les niveaux de la hiérarchie professionnelle, quoique selon des proportions différentes : à mesure que l'on s'éloigne des positions les plus légitimes et d'une définition médicale des tâches, la mobilisation des éléments du « discours faible » tend à augmenter en même temps qu'apparaît plus clairement le rapport social qui se noue dans l'interaction entre patient et professionnel. De ce point de vue, le travail des auxiliaires de puériculture permet de comprendre comment se déclinent les modalités selon lesquelles sont transmis les savoirs entourant le nouveau-né.

L'un des moments au cours desquels s'observent sans doute le mieux, au-delà de l'ethos professionnel, ces logiques sociales de transmission (à l'œuvre dans les interactions) mobilisées par les auxiliaires de puériculture est le moment du bain. La façon dont on doit laver un nouveau-né est fortement investie par l'institution qui la présente à la fois comme une séquence d'actes spécifiques garantissant l'hygiène du nouveauné, et partant sa santé, et comme un moment au cours duquel se donnerait à voir, par son caractère domestique, les façons d'être ordinaires des parents. L'ensemble de ces éléments, à la fois techniques et relationnels, s'inscrit dans un dispositif d'apprentissage explicite. Dans le cadre d'un séjour ordinaire de cinq jours ${ }^{13}$, le nouveau-né va prendre trois à quatre bains : le premier, deux jours après la naissance, est réalisé, dans la majorité des cas, par l'auxiliaire de puériculture ; le second est effectué par la mère assistée, de façon étroite, par la professionnelle qui, les jours suivants, s'effacera plus ou moins selon les acquisitions de la mère. Ce caractère évaluatif n'échappe pas aux parents et contribue, comme on le verra, à renforcer une complexité liée à la tâche autant parce qu'elle demande aux jeunes parents d'interagir pour la première fois avec le nouveau-né (et son corps) que parce qu'elle impose un cadre spécifique marqué par sa technicité et le respect des procédures, dont on peut penser qu'il a dès lors peu à voir avec l'expérience domestique ordinaire (Goffman, 1991). La mâ̂trise des procédures de savonnage, d'immersion, de rinçage, de séchage, d'habillage, de nettoyage des yeux, des oreilles, du nez, du cordon ombilical et de toutes les opérations qui assurent la continuité de la séquence du bain ainsi que les manières de prendre ou de contraindre le corps du nouveau-né tout en gérant, dans le même temps, le matériel nécessaire (serviettes sèches et humides, positionnement des produits, organisation du propre et du sale) permettent ainsi aux auxiliaires de puériculture d'évaluer la possession de savoirs pratiques ad hoc.

Si la répétition quotidienne de la séquence rend possible l'appropriation de sa dimension technique, l'utilisation d'un discours d'accompagnement permet d'en souligner les principes. Les variations dans les pra- 
tiques des auxiliaires paraissent en effet marginales (et renvoient sans doute à des différences entre générations) mais les discours d'accompagnement, quant à eux, sont très différents, y compris chez un même professionnel. Ainsi, alors que le dispositif demeure relativement stable, il fait l'objet de justifications contrastées, voire opposées, qui varient en fonction de l'idée que les professionnels se font de leur interlocutrice. En saisissant dans l'interaction même les mille et un signes (façons de parler, de se tenir, de se vêtir, d'occuper l'espace de la chambre, d'entrer en relation avec le nouveau-né ou le personnel) qui trahissent la position sociale de la mère et, par conséquent, le rapport social qui s'établit alors, les auxiliaires de puériculture vont mobiliser différents registres de discours qui s'adossent à la hiérarchie sociale des savoirs. Sans complètement en rendre raison, elles le signalent d'ailleurs lorsqu'elles disent « s'adapter en fonction de la dame ", " tenir compte de ses capacités ». D'une mère à l'autre (ce qui, dans la quotidienneté du travail dans le service peut signifier d'une chambre à l'autre, d'un instant à l'autre), les discours qui accompagnent le moment du bain vont ainsi d'une explicitation prolixe et techniciste à une quasi-absence de commentaires, en passant par une sorte de relativisation du dispositif au profit du « bien-être ».

Ce sont les mères les plus modestes qui font l'objet des prescriptions les plus explicites. Ces femmes que les professionnelles, entre elles, peuvent nommer les « jeunettes", les « cas soc. " (" cas sociaux"), les « dames d'origine immigrée », font l'objet de « conseils " explicites, de remarques normatives, qui accompagnent la réalisation des différentes étapes du bain. Reprenant le « discours fort » de l'institution à travers des prescriptions techniques renvoyant à des justifications médicales, parfois euphémisées par le recours au "bon sens ", les auxiliaires de puériculture insistent fortement sur la maîtrise de la procédure. Pour ce faire, elles ont le plus souvent recours à de supposées évidences : « II faut que l'eau soit à $37^{\circ} \mathrm{C}$ parce que c'est la température du corps »; "Retirez la serviette pour habiller bébé sinon ses vêtements vont être humides "; "Vous devez bien nettoyer le cordon à chaque change, autrement, ça risque de s'infecter ". La plupart des gestes sont ainsi commentés, y compris ceux qui relèvent des interactions entre la mère et son enfant : elles indiquent ainsi par exemple, lorsque le bébé est immergé, qu'il s'agit d'un bon moment pour lui parler "parce que ça le rassure », «parce que c'est important ». Empruntant parfois la forme scolaire, le discours d'accompagnement peut se muer en interrogations dont on ne sait si elles ont pour but de souligner par la violence qu'elles recèlent l'importance de la phase ou le déficit de maîtrise (supposé) des mères : "Alors, est-ce que vous savez ce que c'est le lait qu'il y a dans les biberons ? "; "Comment, à votre avis, on peut faire pour bien nettoyer le nez de bébé ? »

L'asymétrie du rapport social, favorable à la professionnelle, semble ainsi lui permettre de reprendre à son compte les formes du discours institutionnel d'autorité. À l'opposé, ces manifestations tendent à se réduire lorsque le rapport se renverse, lorsque l'interlocutrice présente les signes d'une appartenance aux catégories les plus socialement élevées. Plus la dissymétrie se renforce, plus le travail des auxiliaires de puériculture apparaît comme relevant des savoirs pratiques, dominé dans la hiérarchie du travail et des professionnels. Leur attitude consiste alors à faire la démonstration des pratiques et, le cas échéant, à répondre aux questions. Les jugements officieux qui circulent alors à propos de ces mères les plus aisées rendent encore plus visible la dimension sociale qui sous-tend cette relation : entre les membres du personnel, ces mères sont souvent décrites comme ne suivant pas les " conseils", telle femme sera désignée comme faisant " un peu ce qu'elle veut " ou comme appartenant à la catégorie indigène des « chieuses ».

Enfin l'ajustement le plus immédiat semble être le fait des femmes se situant dans des positions intermédiaires, décrites comme des "gentilles dames", des « dames sympa », notamment par les sages-femmes et les infirmières, même si les auxiliaires de puériculture, tout en appréciant leur bonne volonté à suivre les " conseils", leur reprochent parfois de " poser beaucoup de questions", de " couper les cheveux en quatre ". Dans leur cas, notamment lorsque la proximité sociale entraîne une certaine forme de sympathie, les auxiliaires de puériculture peuvent s'autoriser à assouplir le dispositif. Les savoirs techniques (masculins) sont ainsi relativisés au profit des savoirs relationnels (féminins), retrouvant le « discours faible » de l'institution. C'est le cas par exemple de cette auxiliaire de puériculture qui, en s'adressant à une mère qu'elle perçoit comme «voulant trop bien faire » (elle pose beaucoup de questions, tremble lorsqu'elle doit agir), lui indique : «Après, nous, on vous montre, et puis, bon, quand vous serez rentrée chez vous, vous allez adapter " et, un peu plus tard : " II faut aussi faire en fonction de comment vous le sentez ». Les logiques qui entourent le bain du nouveau-né se déclinent ainsi par les discours en fonction du rapport social qui se noue, dans l'interaction, entre la professionnelle et la mère. S'il s'agit, à chaque fois, de transmettre les "bonnes » façons de baigner le nouveau-né, les auxi- 
liaires de puériculture, en s'adaptant, modulent les prescriptions de l'institution. Non seulement l'action normative des professionnels de la maternité s'exerce avec d'autant plus d'intensité à mesure qu'elle s'adresse à des mères socialement modestes, mais elle suit la logique de distribution sociale des savoirs en articulant de façon différenciée les " discours fort " et « faible » présents dans l'institution.

Si cette variation de l'action de l'institution sur ces usagers s'observe (ici dans le cas du bain) de façon privilégiée dans le travail des agents situés dans les positions les plus basses de la division du travail, c'est-à-dire aussi dans la hiérarchie des savoirs, on le remarque également dans les autres tâches qui incombent aux auxiliaires de puériculture (alimentation, change) et dans la division des modalités d'interaction des différents corps professionnels à l'intérieur du service. Le contexte de transmission des normes entourant les façons légitimes d'être parent se décline ainsi selon des types d'usagers circonscrits par les professionnels et se module en transposant les hiérarchies qui organisent la division du travail d'encadrement.

\section{RÉSISTANCES ET SOUMISSIONS À L'ACTION INSTITUTIONNELLE}

Si les savoirs transmis dépendent des contextes dans lesquels ils s'énoncent, il reste que l'on peut s'interroger sur la façon dont l'action de l'institution va être reçue par les mères à qui elle s'adresse. L'appropriation des techniques et de leurs principes varie en effet en fonction des types de savoirs qu'ont intériorisés les agents au cours de leur trajectoire antérieure ${ }^{14}$. Selon leur position sociale, les ajustements de l'action de l'institution entraînent une activation des dispositions à agir qui discriminent les pratiques ordinaires des mères.

Ce fondement social des interactions à l'intérieur de l'institution apparaît nettement si l'on considère le cas des femmes qui n'ont que peu de ressources à leur disposition. Dans leur cas, il est impossible, et bien souvent presque impensable, de manifester ouvertement une quelconque résistance face aux prescriptions institutionnelles. En situation, ces femmes font souvent montre d'obéissance, voire d'une certaine docilité alors que le discours des professionnelles devient explicitement normatif et rationnel. Cette apparente soumission à l'institution qui, le plus souvent, passe par une volonté de reproduire à l'identique l'exemple fourni par l'auxiliaire de puériculture et de donner les « bonnes réponses » aux questions posées, peut également engendrer des comportements qui vont à l'encontre de la logique ordinaire. C'est le cas, par exemple, lorsque cette mère baigne son enfant dans une eau à $46^{\circ} \mathrm{C}$ parce que, abandonnant sa pratique ordinaire consistant, sans doute, à apprécier de la main la température de l'eau, elle obéit à l'injonction d'utiliser un thermomètre mais ne parvient pas à le lire correctement (elle n'a pas lu les chiffres en pensant que la juste température se situait au niveau d'un dessin, à vocation décorative, figurant, au côté d'un poisson et d'un petit bateau, le visage d'un enfant souriant).

Toutefois elles font souvent preuve d'une certaine aisance lorsqu'il s'agit de manipuler le nourrisson, contrairement aux femmes situées dans des positions intermédiaires. Chez ces dernières, c'est souvent cette sorte de crainte de mal faire que l'on détecte dans leurs gestes et surtout dans la fréquence de leurs questions. Moins qu'une soumission, c'est une « bonne volonté " parfois anxieuse qu'elles manifestent ainsi et qui conduit les professionnels à tenter de les « rassurer » et à euphémiser leurs injonctions : les discussions prennent plus souvent le ton du " conseil ", de l'échange d'expérience, les auxiliaires de puériculture évoquant, comme confirmation, leurs propres comportements maternels. Si les aspects techniques ou médicaux ne disparaissent pas pour autant, ils sont évoqués de façon plus nuancée, dans le détail : on discute de l'importance du « soin du cordon » en précisant que " ça ne fait pas mal », que «ça peut faire peur » mais qu'« il faut le faire » et que toutes les mères « en sont passées par là ». L'importance accordée au «bienêtre » de la mère et de son enfant, à la « qualité » de leur relation, conduit également à de nombreuses interactions avec le nouveau-né. Le plus souvent, ces mères en viennent à adopter rapidement les pratiques et les principes qui les sous-tendent. C'est avec elles que l'on a constaté, en fin de séjour, à la fois une mise en application méticuleuse des savoirs techniques et la manifestation de manières de faire renvoyant à l'intimité : baisers, caresses, mots d'affection, exprimés sans retenue apparente devant les professionnels ${ }^{15}$ (et les enquêteurs).

Les femmes qui occupent des positions élevées dans la hiérarchie sociale et pour lesquelles les prescriptions des professionnels sont les moins explicites présentent enfin les signes qui témoignent d'une certaine distance par rapport aux prescriptions relationnelles et les plus explicitement éducatives de l'institution, telles cette universitaire (maître de conférence en sciences humaines) qui ne souhaite pas donner le bain à son (second) fils parce qu'elle se dit «fatiguée ", ou 
cette cadre du privé qui, sans s'en apercevoir sans doute, déstabilise la sage-femme en lui déclarant, malgré ses " conseils", qu'elle " n'est pas convaincue " de l'utilité d'une rééducation périnéale réalisée par un kinésithérapeute. Ce type de situations survient d'autant plus fréquemment que le professionnel occupe une position dominée dans la hiérarchie des postes, comme avec les auxiliaires de puériculture, alors qu'il est plus rare lorsque la relation s'équilibre (il est très rare en présence des médecins). On peut donc penser que la transmission des pratiques parentales promues à la maternité est, dans ce cas, assez peu efficiente et que l'action de l'institution se résume à garantir la bonne santé de la femme et de son enfant.

Si l'ajustement réciproque que produit une situation d'interaction se déroulant dans un contexte fortement institutionnalisé explique en partie ces comportements, le fondement social sur lequel il prend appui conduit à considérer également les propriétés sociales des mères. Et notamment, les éléments qui permettent de rendre compte de leur rapport aux institutions et des ressources qu'elles mobilisent participent de l'élaboration de l'idée qu'elles peuvent se faire de ce qu'il convient ou non d'accomplir en tant que parent. Autant que leur volume, c'est le type et l'origine des savoirs dont elles sont porteuses avant leur séjour à la maternité qui contribuent à expliquer leur plus ou moins grande proximité aux normes que l'institution tend à leur transmettre et leur capacité à se les approprier.

Notre enquête retrouve là les analyses développées par Séverine Gojard à propos des pratiques alimentaires parentales : plus les mères disposent d'une position sociale élevée, plus leurs ressources culturelles sont mobilisées pour s'occuper du nouveau-né, et ce de façon d'autant plus autonome par rapport à la maternité que ces ressources sont importantes. À l'opposé, chez les femmes qui en sont le plus dépourvues, on constate une transmission familiale des normes entourant le nourrisson (Gojard, 2000). Pour celles-ci, la docilité souvent constatée dans les observations semble relever davantage d'un rapport de soumission à l'institution hospitalière qui peut dissimuler une stratégie d'évitement des conflits, tout en préservant un certain «quant-à-soi ». C'est ce qu'exprime par exemple cette mère interrogée (18 ans, BEP, au foyer) :

Enquêteur : Et à la maternité, tu as bien aimé le personnel ?

Mère : Nan, tu te sens jugée. C'est dans leur attitude. Tu sais, faut pas les contrarier. Admettons ton chtio y grossit pas, c'est pas de ta faute, mais y vont te juger, te dire que tu lui donnes pas le biberon comme y faut. [...] Mais moi, j'fais comme je veux. Ma mère elle est nourrice, elle a 10 enfants à garder, donc je sais faire avec les bébés. Je fais comme je veux, j'm'en fous de c'qu'y disent.

Cette opposition aux manières de faire promues par la maternité qui, associant le travail maternel à la sphère du privé, prend appui sur une transmission familiale des manières de s'occuper de ses enfants, se voit tempérée à mesure que s'acquiert un petit capital culturel, par l'école bien souvent. C'est ce que I'on constate dans le cas de cette mère rencontrée à la maternité (20 ans, demandeuse d'emploi, baccalauréat professionnel secrétariat, père : menuisier et sans diplôme, conjoint : 21 ans, maçon en CDI) qui, tout en se disant « coachée » par sa belle-sœur dont elle s'occupe parfois des enfants, s'oppose moins qu'elle ne se soumet aux injonctions des professionnels:

Enquêteur : Et alors ici, du coup, on vous explique aussi un peu comment on fait?

Mère : Ouais, ouais mais bon comme là je voulais la faire toute seule la toilette, mais bon elle a voulu m'aider, bon... J'aurais voulu faire toute seule, mais bon c'est rien, je le ferai demain. Ouais, surtout que hier on m'avait déjà aidée quoi.

[...]

Enquêteur : Vous auriez voulu faire le premier bain toute seule?

Mère : Le premier, on me l'a demandé mais non, j'ai pas voulu.

Enquêteur : D'accord. Pourquoi ?

Mère : Ben la peur de mal faire en fait. Puis quand on nous regarde c'est pas pareil. Quand on nous regarde : « II faut faire ci, il faut faire ça ! » Je préfère que... Oui la femme elle l'a fait, je préfère, oui.

\section{CONCLUSION}

La naissance constitue donc bien un moment privilégié d'éducation des mères par différents professionnels qui, en fonction de leur ethos professionnel et de leur place dans l'institution hospitalière, mobilisent des registres de savoirs spécifiques. Les séquences institutionnelles de transmission des pratiques parentales, bien qu'apparemment codifiées et encadrées, si elles se présentent le plus souvent comme des mises en situation des nouveaux parents pour atteindre l'intériorisation d'un ensemble de savoirs techniques, font appel en réalité à des types de pédagogie différents en fonction des caractéristiques sociales des personnes concernées, revêtant une forme de plus en plus explicite et scolaire au fur et à mesure que l'on des- 
cend dans la hiérarchie sociale. Elles ne paraissent produire les effets d'incorporation qu'elles visent que lorsque les femmes auxquelles elles s'adressent reconnaissent la légitimité de l'institution et disposent des ressources sociales et d'une expérience qu'elles vont pouvoir mobiliser ou, tout du moins, au sein desquelles elles vont opérer, de façon pratique, une sélection. Alors, parce qu'une certaine connivence peut s'établir, le « discourt fort » peut progressivement laisser la place au " discourt faible». En revanche, lorsque la distance sociale se fait plus grande, dans un sens ou dans l'autre, les discours des personnels médicaux ou para-médicaux se heurtent à une concurrence avec des savoirs savants (pour les femmes les plus diplômées) ou des savoirs pratiques (dans le cas des femmes des catégories les plus populaires), concurrence qui minore leur efficacité. Dans l'ajustement qui se réalise entre l'institution, par l'intermédiaire notamment des professionnels qui en sont les agents, et l'usager se donnent à voir les effets de positionnement relatif qui révèlent l'existence de styles parentaux socialement distribués et plus ou moins éloignés des normes dominantes portées par l'institution ou le discours public.

Jérôme Camus

Université de Picardie-Jules-Verne, CURAPP

Nathalie Oria

Nathalie.oria@u-picardie.fr

Université de Picardie-Jules-Verne, CURAPP

\section{NOTES}

1 Cf. par exemple, pour le cas des jeunes enfants accueillis dans les services de PMI, le travail de Serre (1998).

2 On estime que la part des naissances se déroulant dans un établissement spécialisé est aujourd'hui, en France, de 99 \% (selon le rapport pour la MIRE, voir Akrich, Develay, Naiditch et al. 2000). Cette médicalisation de l'accouchement s'est déroulée 2000). Cette médicalisation de l'accouchement s'est déroulée au cours de la seconde moitié du xxe siècle, la part des accouchements à domicile passant dans les années cinquante d'environ $50 \%$ à moins d' $1 \%$ au début des années quatre-vingt
(Naulleau, 1982). On peut noter que, si ce mouvement est caractéristique des pays européens, son intensité est spécifique à la France, les Pays-Bas présentant le taux d'accouchement à domicile le plus élevé (environ $30 \%$ ).

3 Sur l'histoire de l'encadrement des parents, et notamment des mères, cf. Garcia (2011). Pour une illustration récente et retentissante de ces débats, voir les échanges parfois vifs qui ont entouré la publication du dernier ouvrage d'Élisabeth Badinter (2010)

4 Les maternités en France sont classées en fonction de leur équipement technique : les maternités de niveau 1 accueillent les accouchements classiques et celles de niveau 3 , situées dans les centres hospitaliers universitaires, sont dotées de services de néonatologie et de réanimation néonatale. Les femmes ayant une grossesse dite " à risque " sont automatiquement orientées vers les maternités de niveau 3.

5 Cette enquête s'inscrit dans le projet «PIC-ENFAN : les conditions sociales et politiques de la naissance en Picardie et en France », financé par le conseil régional de Picardie.

6 II apparaît que le suivi de la grossesse fait intervenir plusieurs professionnels de santé : pendant cette période, $65 \%$ des femmes consultent un gynécologue en maternité $(45 \%$ hors maternité), $26 \%$ ont eu au moins une consultation par une sage-femme en maternité (5\% par une sage-femme libérale). Le rôle du médecin généraliste ne doit pas être sous-estimé, notamment en début de grossesse, puisque $24 \%$ des déclarations de grossesse (intervenant avant la fin du troisième mois de grossesse) sont effectuées par eux. II reste néanmoins que l'institution hospitalière occupe une place très importante, qui se renforce à mesure qu'avance la grossesse : la part des femmes sement dans lequel elles ont accouché est de $33 \%$, mais seules
$8 \%$ d'entres elles n'ont eu aucun contact avec celui-ci avant la naissance (voir Blondel, Supernant, Mazaubrun et al., 2005).

7 II s'agit d'un site Internet disponible à l'adresse : <www.lelivrebleu.fr> (consulté le 30 août 2011).

8 Cette préparation à la naissance est suivie par $66 \%$ des primipares et $25 \%$ des multipares (Blondel, Supernant, Mazaubrun et al., 2005).

9 Ce référentiel de formation est disponible en ligne : <http://www. ordre-sages-femmes.fr/NET/fr/document//2/exercice_de_la_ profession/comment devenir_sagesfemmes_/la_formation/ index.htm> (consulté le 30 août 2011).

10 Sur l'histoire de cette différenciation entre médecin et sagefemme à travers laquelle on retrouve les oppositions entre savoirs techniques et savoirs relationnels, mais aussi, et plus largement, entre sphère publique et privée, masculin et féminin, cf. Gélis (1988) et Schlumbohm (2002).

$11 \mathrm{Au}$ moins idéalement, car les contraintes liées aux logiques managériales viennent fréquemment s'y opposer.

12 Cette différenciation, qui suit la hiérarchie professionnelle, n'est pas sans rappeler ce que décrit Paillet à propos de l'éthique en réanimation néonatale (Paillet, 2007).

13 Cette durée ordinaire, constatée dans les deux maternités enquêtées, est légèrement supérieure à la moyenne nationale (4,4 jours en 2010 pour un « accouchement normal », selon l'Assurance maladie, voir par exemple : <http://www.newspress.fr/ Communique_FR_227613 854.aspx>, consulté le 30 août 2011).

14 Tout en observant le travail quotidien des auxiliaires de puériculture et de sages-femmes, on a pu recueillir des informations minimales sur les mères observées: profession (et parfois celle du conjoint), nombre d'enfants, nom et rang de naissance du nouveau-né, type d'alimentation (au biberon ou au sein).

15 Il faut préciser que, dans certains cas, ces injonctions à l'expression d'une nature maternelle plongent les mères dans une grande perplexité. On pense par exemple à cette sage-femme venant d'avoir son premier enfant et qui, selon ses collègues, " n'arrêtait pas de pleurer " ou cette infirmière qui explique au cours de l'entretien que, malgré son assurance dans la réalisation des soins, elle ne " ressent rien ", ne comprend pas ce que peut bien vouloir dire "faire comme on le sent " et en vient à en éprouver une « vraie panique ». 


\section{BIBLIOGRAPHIE}

AKRICH M., DEVELAY A., NAIDITCH M. \& PASVEER B. (2000). Dispositifs d'offres de soins obstétrico-pédiatriques : filières, trajectoires, usagers. Cahiers de recherches de la MIRE, $n^{\circ} 8$, p. 3-8.

ARBORIO A.-M. (2002). « Les aides-soignantes, catégorie d'assistance aux professionnels : une impossible professionnalisation ? "Santé publique et sciences sociales, $n^{\circ} 8-9$, p. 203-216.

BADINTER É. (2010). Le conflit. La femme et la mère. Paris : Flammarion.

BLONDEL B., SUPERNANT K., MAZAUBRUN C. du \& BRÉART G. (2005). Enquête nationale périnatale 2003. Situation en 2003 et évolution depuis 1998. Paris : Ministère des Solidarités, de la Santé et de la Famille et Institut national de la santé et de la recherche médicale. Disponible sur Internet à l'adresse : <http:// www.sante.gouv. fr/resultats-de-I-enquete-nationaleperinatale-2003.html> (consulté le 30 août 2011).

BOLTANSKI L. (1969). Prime éducation et morale de classe. Paris : Éd. de l'EHESS.

BONNÉRY S. (2007). Comprendre l'échec scolaire. Élèves en difficultés et dispositifs pédagogiques. Paris : La Dispute.

BOURDIEU P. (1997). Les méditations pascaliennes. Paris : Éd. du Seuil.

CARRIÉRE C (2008). Se faire parents à l'hôpital. Intéractions et co-élaborations des normes de parentalité dans un service de suivi de grossesse à haut risque. Dossier d'étude ${ }^{\circ} 101$, Paris. CNAF.

CICOUREL A. (2002). Le raisonnement médical. Paris : Éd. du Seuil.

DAGNAUD M. \& MEHL D. (1988). "Les gynécologues : une profession influente". Sociologie du travail, vol. 30, $n^{\circ} 2$, p. 287-300.

DELAISI DE PARSEVAL G. \& LALLEMAND S. (1979). L'art d'accommoder les bébés. 100 ans de recettes françaises de puériculture. Paris : Éd. du Seuil.

DELBOS G. \& JORION P. (1990). La transmission des savoirs. Paris : Éd. de la MSH.

DONZELOT J. (1977). La police des familles. Paris : Éd. de Minuit.

DURIF-BRUCKERT C. (2006). " Le transfert des savoirs de santé vers le grand public : complexité méthodologique et enjeux psychosociaux ". In V. Haas (dir.), Les savoirs du quotidien, transmissions, appropriations, représentations. Rennes: Presses universitaires de Rennes.

GARCIA S. (2011). Mères sous influence. De la cause des femmes à la cause des enfants. Paris : La Découverte.

GÉLIS J. (1988). La sage-femme ou le médecin. Une nouvelle conception de la vie. Paris : Fayard.

GOFFMAN E. (1991). Les cadres de l'expérience. Paris : Éd. de Minuit.
GOJARD S. (1998). Nourrir son enfant : une question d'éducation. Normes savantes, usages populaires et expérience familiale. Thèse de doctorat, sociologie, EHESS.

GOJARD S. (2000). " L'alimentation dans la prime enfance. Diffusion et réception des normes de puériculture ". Revue française de sociologie, vol. 41, $n^{\circ} 3$, p. 475-512.

GRIGNON C. (1971). L'ordre des choses. Les fonctions sociales de l'enseignement technique. Paris : Éd. de Minuit.

JACQUES B. (2007). Sociologie de l'accouchement. Paris : PUF.

JAISSON M. (2002). « La mort aurait-elle mauvais genre ? La structure des spécialités médicales à l'épreuve de la morphologie sociale ". Actes de la recherche en sciences sociales, $n^{\circ} 143$, p. 44-52.

KELLERHALS J., MONTANDON C., GILBERT R. \& SARDI M (1992). " Le style éducatif des parents et l'estime de soi des adolescents ". Revue française de sociologie, vol. 33, n 3 , p. 313-333.

MAUGER G. \& POLIAK F. (2000). «Lectures : masculin/féminin ». Regards sociologiques, $n^{\circ} 19$, p. 115-140.

NAULLEAU E. (1982). "L'accouchement à domicile ». Économie et statistique, $\mathrm{n}^{\circ} 148$, p. 53-55.

PAILLET A. (2007). Sauver la vie, donner la mort. Une sociologie de l'éthique en réanimation néonatale. Paris : La Dispute.

POURTOIS J.-P., DESMET H. \& LAHAYE W. (2004). "Connaissances et pratiques en éducation familiale et parentale 》. Enfance, familles, générations, $\mathrm{n}^{\circ} 1$, p. 22-35.

POURTOIS J.-P., FORGIONE A., DESMET H. et al. (1984). Éduquer les parents ou comment stimuler la compétence en éducation. Bruxelles : Labor.

SCHLUMBOHM J. (2002). "Comment l'obstétrique est devenue une science. La maternité de l'université de Göttingen, 1751-1830 ». Actes de la recherche en sciences sociales, $n^{\circ} 143$, p. 18-30.

SCHWARTZ O. (1990). Le monde privé des ouvriers. Hommes et femmes du Nord. Paris : PUF.

SERRE D. (1998). «Le "bébé superbe". La construction de la déviance corporelle par les professionnel(le)s de la petite enfance ". Sociétés contemporaines, $n^{\circ} 31$, p. 107-127.

THIN D. (1998). Quartiers populaires. L'école et les familles. Lyon : Presses universitaires de Lyon.

TILLARD B. (2003). Des familles face à la naissance. Paris : L'Harmattan.

WILLIS P. (1978). "L'école des ouvriers ". Actes de la recherche en sciences sociales, $\mathrm{n}^{\circ} 24$, p. 50-61. 